\title{
Discrete phase numerical analysis of servo valve nozzle
}

\author{
Junye $\mathrm{Li}^{1}$, Ningning $\mathrm{Su}^{2}$, Zengwei Zhou ${ }^{3}$, Lili Wei ${ }^{4}$, Xinming Zhang \\ College of Mechanical and Electric Engineering, Changchun University of Science and Technology, \\ Changchun, China \\ ${ }^{1}$ Corresponding author

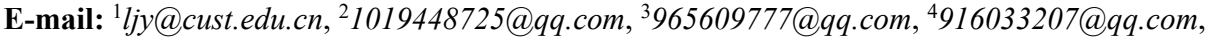 \\ 5xxm@cust.edu.cn
}

Received 23 October 2017; accepted 30 October 2017 DOI https://doi.org/10.21595/vp.2017.19320

Check for updates

\begin{abstract}
In order to study the polishing quality of abrasive flow, The FLUENT15.0 software is used as the platform, taking the way that LES (Large-eddy simulation) model is combined with the Mixture model, the numerical simulation of Grain Flow Processing was carried out by regarding the servo valve nozzle as the research object. The results turn out that with the increase of the inlet speed, the flow state of the abrasive flow is more disorderly, and there is a dramatically improvement in dynamic pressure, which indicates that the collision and friction between the abrasive grains and the wall is more intense. In addition, the vortices and turbulence are more apparent, which are conducive to improving the polishing efficiency of abrasive flow so that achieve precision machining.
\end{abstract}

Keywords: abrasive flow technology, servo valve nozzle, precision machining.

\section{Introduction}

The servo valve core nozzle is the key part of the servo valve, whose operation performance will be directly affected by its surface quality. When there are burrs or not smooth enough at the inside wall of the nozzle [1-3], it is likely to cause scattering and spirals or cause the nozzle body to clog once the fluid flows. As a consequent, the entire servo valve entire performance will be subjected to serious damage. It is fairly significant to carry out the precision finishing of the nozzle so as to improve the quality of the nozzle small hole $[4,5]$.

Jose Cherian [6] et al. studied the relationship between the fatigue properties of the material and the surface finish. The process parameters such as pressure, abrasive concentration and grid size were selected as the object of study, and the influence of process parameters on the fatigue intensity was discussed in the abrasive flow polishing process. Li Junye $[3,7,8]$ et al. designed a kind of abrasive grain polishing device to study the precision problem of the common-rail micro-hole. The ideal scheme of the abrasive flow processing was obtained through the simulation study of the abrasive flow of the common rail. The experimental results provide a theoretical basis for the optimization of the polishing process parameters of the abrasive grain. The micro-fabrication mechanism of polished non-linear tube parts was studied and analyzed by taking the common-rail as an object. The influence of key process parameters on the surface roughness of the part was tested by means of the grinding solution.

\section{Model calculation and boundary conditions}

\subsection{Boundary conditions}

In order to reveal the effect of grinding fluid flow in practice, the servo valve nozzle was used as the research object, and the FLUENT15.0 as the calculation platform, LES turbulence model was combined with the Mixture multiphase flow model to conduct numerical simulation. The main components of the abrasive flow are silicon carbide and hydraulic oil. The solver was executed by use of 3D double precision, velocity-inlet and pressure-out. The number of iterations is set up for 500 steps and 212 steps converge. 


\subsection{Mesh division}

Meshing division is the most critical part of the numerical simulation process, which has a great influence on the simulation results. So, it is necessary to give enough attention to the quality of the grid division. The grinding fluid flow is in-compressible fluid, and the flow channel is cylindrical, the three -dimensional model was built in order to be close to the actual and easy to observe, the mesh division of the flow channel is shown in Fig. 1.

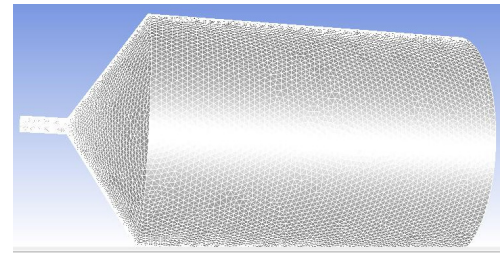

Fig. 1. Meshing division

\section{Analysis of numerical simulation of the discrete phase}

Abrasive flow processing as a fluid processing, which can be used to process a variety of tiny holes and complex surfaces, and achieve the micro-removal to achieve the purpose of precision polishing. As the servo valve nozzle size has a very small size, so the inlet speed must be carefully selected or there will appear two situations that too much speed may damage the workpiece and too small and cannot achieve the effect of precision machining. By referring the relevant data, the inlet speed of $5 \mathrm{~m} / \mathrm{s}, 10 \mathrm{~m} / \mathrm{s}, 15 \mathrm{~m} / \mathrm{s}$ and $20 \mathrm{~m} / \mathrm{s}$ was choosing respectively to perform numerical simulation of the servo valve nozzle. The dynamic pressure and vortex distribution were analyzed at different inlet velocity so that verify the effectiveness and practicability of abrasive grain flow processing.

Dynamic pressure is an important factor to reflect the removal rate of material. The greater the pressure, the more intense the abrasive flow and the wall friction, the better the material removal effect. The dynamic pressure diagram is shown in Fig. 2.

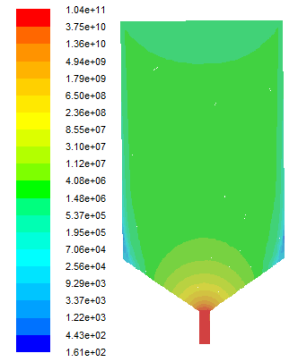

a) Inlet velocity $=5 \mathrm{~m} / \mathrm{s}$
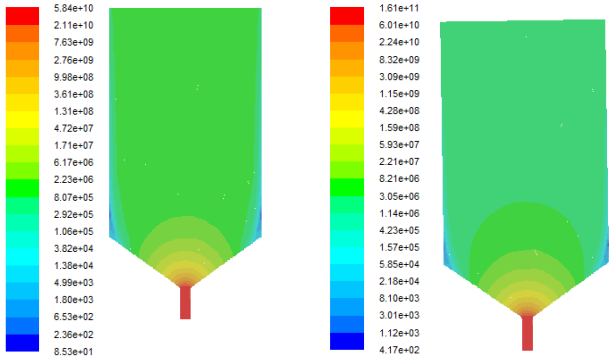

b) Inlet velocity $=10 \mathrm{~m} / \mathrm{s} \quad$ c) Inlet velocity $=15 \mathrm{~m} / \mathrm{s}$

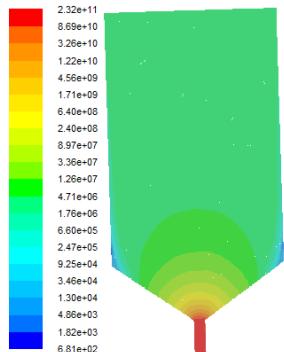

d) Inlet velocity $=20 \mathrm{~m} / \mathrm{s}$

Fig. 2. The dynamic pressure graph at different inlet velocity

It can be seen from Fig. 2 that with the gradual increase in the inlet speed, the dynamic pressure is gradually increased. When the fluid does not reach the top of the nozzle yet, the dynamic pressure is substantially unchanged, and even a little less as the velocity increases. Because in this section, the inlet radius is large, when the abrasive flow enters the runner, there is a small collision and intersection between the fluids, which will produce a reduction for turbulent intensity, so is the dynamic pressure. When the fluid reaches the top of the servo valve nozzle, due to the sudden decrease of the cross-sectional area, as a result, there is a sharp increase in flow speed. At the same time, there appear a severe impact and friction between fluid and fluid or fluid and wall, therefore, the dynamic pressure also increases. The analysis reveals that abrasive flow machining has a great 
polishing effect and can be very good to achieve the role of deburring.

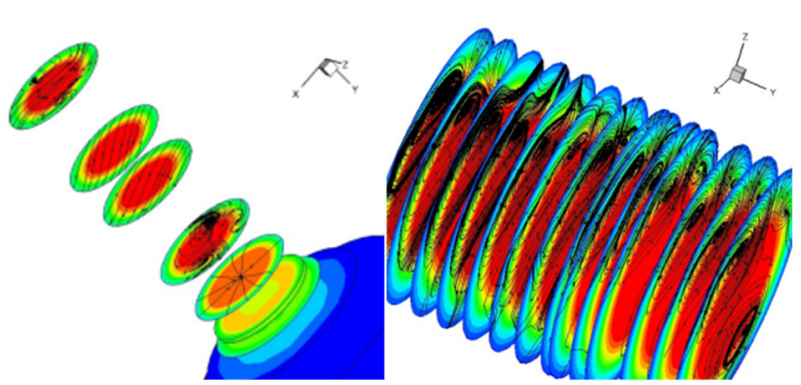

a) Partial magnification at the nozzle

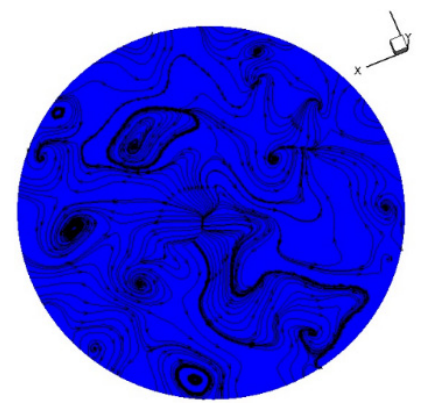

b) Nozzle vortices at the intersection

Fig. 3. Vortex distribution

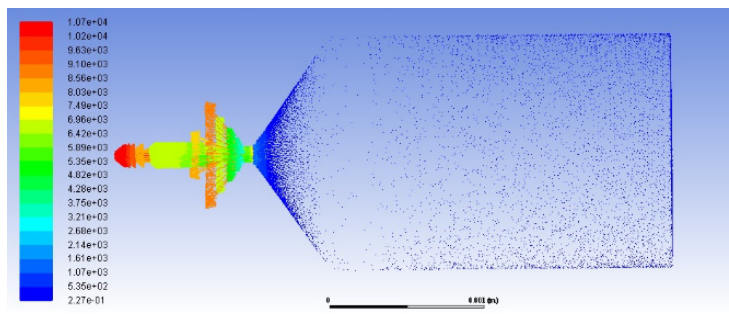

a)

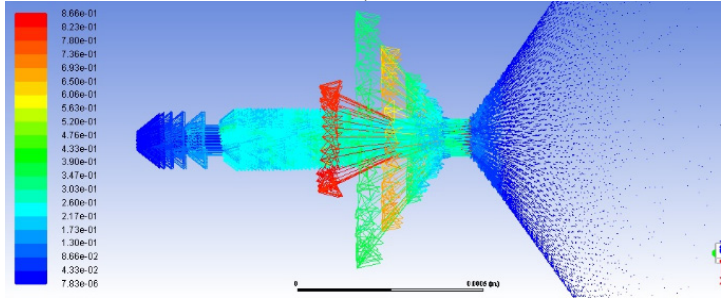

b)

Fig. 4. Speed vector graph

It can be detected that in the same section, the intensity of the vortex that the abrasive flow affiliated varied with the distance the wall to the vortex center. Consequently, the wall of the polishing effect is bound to be different. We can draw a conclusion from Fig. 3 that the vortex is mainly distributed in the cross-section. By comparing with the state of the vortex can be known that before the fluid flows into intersection, most of the collision will happen so that result vortex as a result of the sudden changing of the diameter, and the shape of the vortex is irregular. The regular vortices appear mainly in the back of the thin pipe, where the vortices are continuous and stable, with a certain shape, rather than a mess at the interface with the thick pipe. The change of the steady vortex becomes more intense as the velocity increases, and carry more energy. As the abrasive grain squeeze grinding effect, there will be a larger fillet at the intersection of the greater the speed, which can effectively reduce the high pulse fatigue stress to improve fatigue strength. With the abrasive flow processing method can get the ideal surface quality and can also enhance the reliability of parts to extend the life.

\section{Conclusions}

The numerical simulation of the servo valve nozzle was carried out by using FLUNET15.0 as the calculation platform by establishing the LES model and the mixture model. The dynamic pressure and the vortex distribution at different inlet velocities were analyzed, which provides a 
theoretical basis for abrasive flow process.

The numerical results show that the dynamic pressure increases with the increase of the inlet velocity, and the larger squeezing and friction with the wall will be produced by the abrasive flow, which aggravates the collision between the fluid and the wall. Moreover, the material removal rate will get a corresponding improvement, so does the polishing quality. Through the vortex distribution state can be found that in the nozzle, the vortex distribution is relatively uniform, with a regular shape, which can improve the uniformity of abrasive grain polishing.

\section{Acknowledgements}

The authors would like to thank the National Natural Science Foundation of China No. NSFC 51206011, Jilin Province Science and Technology Development Program of Jilin Province No. 20160101270JC and No. 20170204064GX, Project of Education Department of Jilin Province No. 2016386.

\section{References}

[1] Shiming J., Chen L., Dapeng T., et al. Study on machinability of softness abrasive flow based on Preston equation. Journal of Mechanical Engineering, Vol. 47, Issue 17, 2011, p. 156-163.

[2] Yuanqiang T., Yi L., Yong S. On the model and pressure simulation of solid- fluid two phase flow for abrasive flow machining. China Mechanical Engineering, Vol. 19, Issue 4, 2008, p. 439-441.

[3] Junye L., Zemin Q., Zhaojun et al. Y. Influence of abrasive concentration on the processing quality of abrasive flow in mesoscopic scale. Journal of Jilin University (Engineering and Technology Edition), Vol. 47, Issue 3, 2017, p. 837-843.

[4] Jinfu D., Runzhi L., Kehua Z., et al. Micro cutting mechanism of abrasive flow precision machining. Optics and Precision Engineering, Vol. 22, Issue 12, 2014, p. 3324-3331.

[5] Bordatchev E. V., Hafiz A. M. K., Tutunea Fatan O.-R. Performance of laser polishing in finishing of metallic surfaces. The International Journal of Advanced Manufacturing Technology, Vol. 73, Issue 1, 2014, p. 35-52.

[6] Jose C., Jeoju M. I. Fatigue performance in abrasive flow machining. Dynamics of Machines and Mechanisms, Industrial Research, Vol. 592, Issue 594, 2014, p. 354-362.

[7] Junye L., Weina L., Lifeng Y., et al. Micro-hole of the common-rail abrasive flow machining equipment design and numerical simulation. Machinery Design and Manufacture, Vol. 10, 2010, p. 54-56.

[8] Junye L., Jinglei H., Kun D. Analysis to erosive wear of abrasive flow polishing servo valve core nozzle. Optical and Precision Engineering, Vol. 25, Issue 7, 2017, p. 1857-1865. 